\title{
Country-level correlates of e-cigarette use in the European Union
}

\author{
Giuseppe La Torre $\cdot$ Daniele Mipatrini
}

Received: 30 June 2015/Revised: 15 January 2016/Accepted: 20 January 2016

(C) Swiss School of Public Health (SSPH+) 2016

\begin{abstract}
Objectives The aim of this study was to assess the relationship between the prevalence of e-cigarette use and country-level factors across 28 European countries.

Methods The study objectives were addressed in an ecological design in which both exposures and outcomes were measured at the country level. Data from the Eurobarometer Report, the Eurostat database and the WHO observatory were analyzed. Bivariate and multivariate analyses were performed considering the rates of current and ever smokers of e-cigarettes as dependent variables, and socio-economic factors, health status and policies against tobacco as independent variables.

Results Both the rate of current smokers and ever smokers of e-cigarette were positively associated to the offer of help to quit tobacco use $(P<0.01 ; P=0.04)$ and to the raise of taxes on tobacco $(P=0.01 ; P=0.01)$. The warn on dangers of tobacco negatively correlated with the rate of e-cigarette current smokers. The rate of current e-cigarette smokers correlated with the rate of current smokers and with national Gross Domestic Product, while
\end{abstract}

This article is part of the special issue "Electronic Cigarettes and Public Health".

Electronic supplementary material The online version of this article (doi:10.1007/s00038-016-0792-1) contains supplementary material, which is available to authorized users.

G. La Torre $\cdot$ D. Mipatrini $(\bowtie)$

Department of Public Health and Infectious diseases, Sapienza

University of Rome, Piazzale Aldo Moro 5, 00185 Rome, Italy

e-mail: daniele.mipatrini@uniroma1.it

G. La Torre

e-mail: giuseppe.latorre@uniroma1.it the rate of ever e-cigarette smokers did not correlate with any socio-economic factor.

Conclusions Our analysis suggests that both policy and non-policy factors are associated with the geographical variability seen in the prevalence of e-cigarette use. Policies against the consumption of conventional tobacco products may lead to an increase of e-cigarette smokers.

Keywords e-Cigarette $\cdot$ Europe $\cdot$ Smoking cessation $\cdot$ Tobacco policy

\section{Introduction}

Electronic cigarettes (e-cigarettes) deliver a nicotine-containing aerosol to users by heating a solution containing propylene glycol or glycerol, nicotine and flavoring agents. Use of e-cigarettes since their creation in the early $2000 \mathrm{~s}$ by Hon Lik, a Chinese pharmacist, has been widespread (Grana et al. 2013). A recent systematic review (Gualano et al. 2014) suggests that e-cigarette use may help reduce the number of cigarettes smoked as well as nicotine withdrawal symptoms. However, current knowledge on possible long-term adverse effects of e-cigarette use remains limited since most studies assess use over short time periods only. As discussed by Famele et al. (2015), there may be harmful substances produced during the heating process, and in-depth chemical analyses of the aerosol are needed to fully assess the chemical consequences of e-cigarette use. Moreover, there is evidence that dual use of conventional and e-cigarettes does not help smokers quit or reduce smoking (Manzoli et al. 2015).

Because of the lack of knowledge on the effectiveness and risks of e-cigarette use, policy makers often adopt a cautious approach (Smith 2014; Kadowaki et al. 2015). 
The American Heart Association (AHA) in a recent policy statement published in Circulation (Bhatnagar et al. 2014), considers e-cigarettes containing nicotine as tobacco products. The direct corollary is that their regulation should comply or align with existing laws on the use and marketing of tobacco products. In this statement, the AHA strongly supported laws and regulations that prohibit the sale and marketing of e-cigarettes to young people.

In the European Union, the Tobacco Products Directive (2014/40/EU) establishes rules governing the manufacture and sale of tobacco and related products including e-cigarettes. The Directive sets out safety and quality requirements for tobacco consumers, and e-cigarette users in particular. However, this Directive may not be easy to implement at the national level.

In the absence of evidence-based policy, e-cigarette use must be closely monitored. Vardavas et al. (2014), using data from the 2012 Eurobarometer 385 Survey, reported that e-cigarette users are more likely to be younger, current smokers, or past-year quit attempters. However, their analysis was conducted at the individual level, and no insight was obtained on the possible influence of countrylevel factors including tobacco control policies. The aim of this study was therefore to assess the association between the prevalence of e-cigarette use and country-level factors across 28 European countries.

\section{Methods}

The study objectives were addressed in an ecological design in which both exposures and outcomes were measured at the country level. Specifically, we examined the association between the prevalence of current and ever e-cigarette use in 28 European Union countries and selected country-level factors.

\section{Study variables}

Data on the prevalence of current and ever e-cigarette use were drawn from the Eurobarometer "Attitudes of Europeans towards tobacco and electronic cigarettes" report published in May 2015 (European Commission 2015). This report summarizes data collected in 1000 interviews conducted in each of the 28 countries of the European Union in 2014. We defined "current e-cigarette users" as those who answered "yes" to the question: "Are you currently smoking electronic cigarettes or similar devices?" "Ever e-cigarette users" included current smokers, former smokers and participants who had tried e-cigarettes in the past, but no longer used them. Data on the prevalence of e-cigarette users and conventional cigarette smokers are provided in Online Resource 1.
Country-level factors investigated as correlates of e-cigarette use included both tobacco policy variables and selected non-policy variables. Non-policy variables included the prevalence of conventional cigarette smoking, economic status, educational attainment and health status. Data on the prevalence of current and former conventional cigarette smoking were drawn from the Eurobarometer report (European Commission 2015). Economic status was measured using data on two indicators [i.e., Gross Domestic Product (GDP) per person, unemployment rate] drawn from the 2014 Eurostat database (Database-Eurostat 2015). Educational attainment was measured by the prevalence of persons age 18 years currently in school as reported in the Eurostat database (Database-Eurostat 2015). Two indicators were used to measure health status: Healthy Life Years expectancy at birth stratified by sex, and self-reported unmet need for medical examination or treatment. Data for these variables were drawn from the 2014 Eurostat database (Database-Eurostat 2015), and are provided in Online Resource 1.

To measure country-level tobacco control policies, we drew data on policies in Europe in 2014 from the WHO Global Health Observatory Data Repository (Global Health Observatory Data Repository 2015). Seven policies were retained: presence of an active monitoring system of smokers indicators, the active protection from tobacco smoke (ban on smoking in public settings), help to quit tobacco smoke, the warn on dangers of tobacco (health warnings on tobacco products), the presence of anti-tobacco mass media campaigns, the enforcement of bans on tobacco advertising, and the raise of taxes in the retail price of tobacco products. Each policy in each country was scored according to a "best practice ranking" of the policy. Countries were scored 1 if data on policy implementation at the national level were not available or if the anti-tobacco policy was not present in the country, 2-4 if the country was active in the area, but did not implement or only partially implemented the best practice, and 5 if the country fully implemented the best policy in the area. For example, a score of 5 on the policy offering help to quit tobacco smoke indicated that the country fully implemented a national quit line, and both nicotine replacement therapy and cessation services available in hospitals or the community were cost covered. Detailed explanations of the seven indicators and their scoring are presented in Table 1. Data are available in Online Resource 1.

\section{Data analysis}

Spearman rank correlation coefficients were computed to describe the correlation between current and ever e-cigarette use and each potential correlate of interest. We then undertook two multivariable analyses for each outcome 


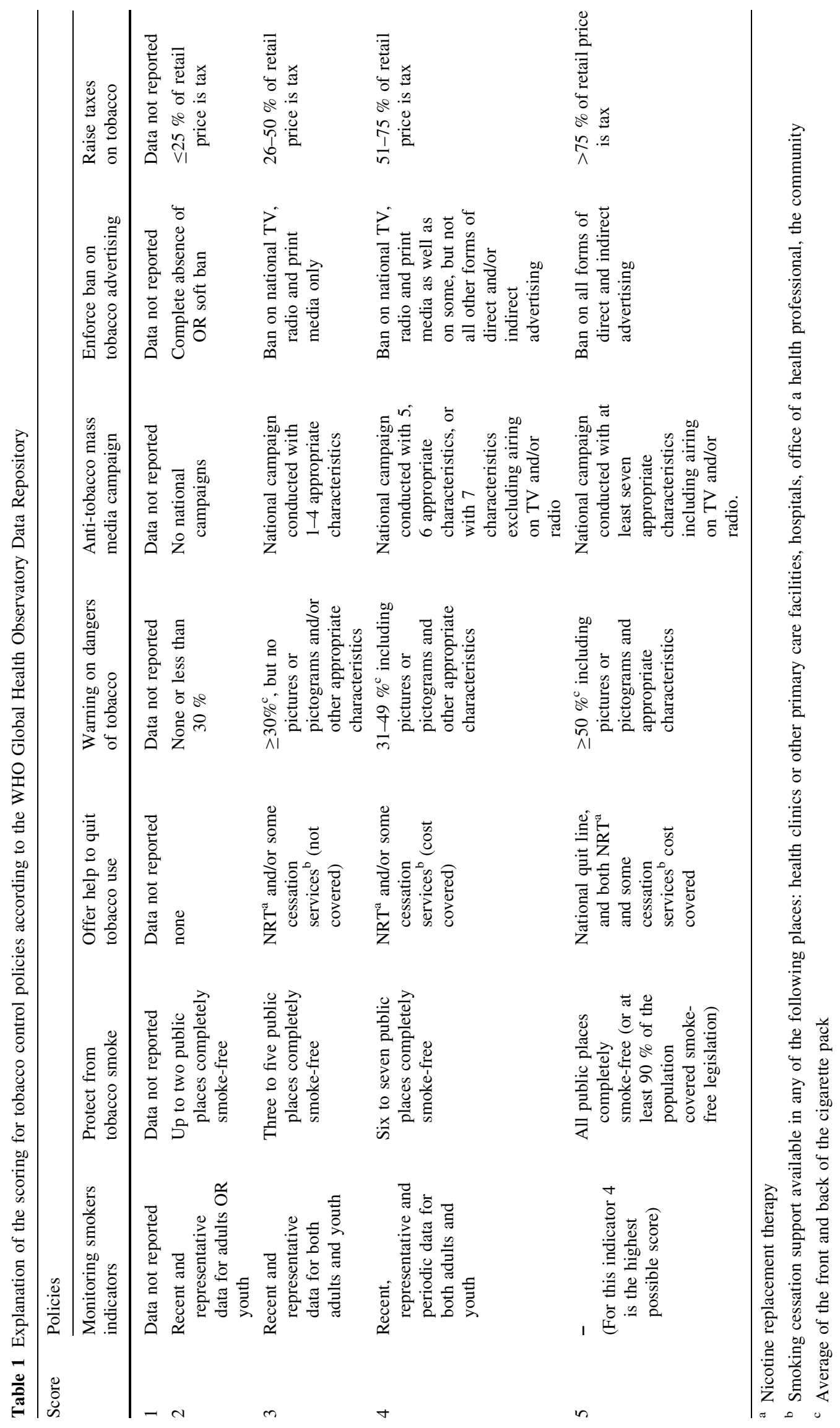


(current e-cigarette use; ever e-cigarette use) adopting a stepwise approach with backwards elimination. The first model included only the non-policy potential correlates. To avoid multicollinearity in this model, we selected only one indicator for each of use of conventional cigarettes (i.e., either current or former conventional cigarette smoking), economic status (GDP per person or unemployment rate) and health status (Healthy Life Years expectancy at birth in males and females, or unmet need for medical examination or treatment).

The second model included all the policy variables except presence of an active monitoring system of tobacco consumption, which was viewed as a method to monitor changes due to the other six policies. The analyses of the first two models were performed using a stepwise approach with backwards elimination. A third model included variables retained in the last step of the backward elimination in the two previous analyses plus one indicator of use of conventional cigarettes and GDP per person if these were not retained in the last step of the backwards elimination. Finally, we produced a model for each outcome with five covariates.

Statistical significance was set at $p<0.05$. The analysis was conducted using SPSS for Windows, release 22.0

\section{Results}

According to the 2014 Eurobarometer report, the average prevalence of current e-cigarette use was $2 \%$ across European countries. The lowest prevalence was less than
$1 \%$ in Hungary, Lithuania, Malta, Romania, Slovenia and Sweden while the highest prevalence was $4 \%$ in the UK. The prevalence of ever e-cigarette use was higher-12\% of Europeans reported trying e-cigarettes. Portugal reported the lowest prevalence $(6 \%)$ while France reported the highest prevalence (21\%) (European Commission 2015).

Table 2 summarizes the correlations between the country-level potential correlates and the prevalence of current and ever e-cigarette use across the 28 European Union countries.

The only variable that was statistically significantly correlated with the prevalence of current e-cigarette use was the best practice ranking the offer of help to quit tobacco smoke. None of the other potential correlates investigated were statistically significantly correlated with the prevalence of current or ever e-cigarette use at $p<0.05$.

Multivariately, several policy variables were statistically significantly associated with the prevalence of current and ever e-cigarette use (Table 3 ).

Specifically, in the third model which included both non-policy and policy variables, the prevalence of current e-cigarette use was associated with the best practice rankings of the offer of help to quit tobacco consumption $(\beta=0.68 ; P<0.01)$ and the raise of taxes on tobacco ( $\beta=0.39 ; P=0.01)$. The best practice ranking of the warn on dangers of tobacco (health warnings on tobacco products) was negatively associated with the prevalence of current e-cigarette use $(\beta=-0.38 ; P=0.04)$. GDP per person $(\beta=0.48 ; P=0.01)$ and current use of conventional cigarettes $(\beta=0.35 ; \quad P=0.04)$ were both associated with the prevalence of current e-cigarette use.

Table 2 Correlation between potential correlates of the prevalence of ever and current e-cigarette use among European Union countries in 2014

\begin{tabular}{|c|c|c|c|c|}
\hline & \multicolumn{2}{|c|}{ Current e-cigarette use } & \multicolumn{2}{|c|}{ Ever e-cigarette use } \\
\hline & $\begin{array}{l}\text { Spearman } \\
\text { correlation }\end{array}$ & $P$ & $\begin{array}{l}\text { Spearman } \\
\text { correlation }\end{array}$ & $P$ \\
\hline Prevalence of conventional cigarette smokers & 0.12 & 0.56 & 0.01 & 0.99 \\
\hline Prevalence of conventional cigarette ex-smokers & 0.12 & 0.53 & 0.25 & 0.19 \\
\hline Gross Domestic Product (GDP) per person & 0.35 & 0.07 & 0.16 & 0.43 \\
\hline Unemployment rate & 0.16 & 0.43 & -0.06 & 0.75 \\
\hline 18 year-olds enrolled in education & -0.07 & 0.72 & -0.08 & 0.68 \\
\hline Female life expectancy in good health & 0.09 & 0.65 & 0.25 & 0.20 \\
\hline Male life expectancy in good health & 0.15 & 0.45 & 0.20 & 0.31 \\
\hline Unmet medical needs & -0.12 & 0.56 & 0.02 & 0.92 \\
\hline Monitoring smokers indicators & -0.01 & 0.94 & 0.01 & 1.00 \\
\hline Protect from tobacco smoke & 0.01 & 0.95 & 0.03 & 0.90 \\
\hline Offer help to quit tobacco use & 0.42 & 0.03 & 0.35 & 0.06 \\
\hline Warn about the dangers of tobacco & 0.05 & 0.80 & 0.18 & 0.37 \\
\hline Mass media campaigns against tobacco & -0.01 & 0.95 & 0.24 & 0.22 \\
\hline Enforce bans on tobacco advertising & -0.05 & 0.80 & -0.07 & 0.72 \\
\hline Raise taxes on tobacco products & 0.25 & 0.19 & 0.34 & 0.08 \\
\hline
\end{tabular}


Table 3 Beta coefficients from multivariate analyses of the determinants of the prevalence of current and ever e-cigarette use among European Union countries in 2014

\begin{tabular}{|c|c|c|c|c|c|c|}
\hline & \multicolumn{3}{|c|}{ Current e-cigarette use } & \multicolumn{3}{|c|}{ Ever e-cigarette use } \\
\hline & Beta & $P$ & Adjusted $R^{2}$ & Beta & $P$ & Adjusted $R^{2}$ \\
\hline \multicolumn{7}{|l|}{ Model 1: socio-economic factors } \\
\hline Conventional cigarette smokers & 0.37 & 0.09 & 0.19 & a & a & 0.01 \\
\hline Gross Domestic Product (GDP) per person & 0.57 & 0.01 & & a & a & \\
\hline \multicolumn{7}{|l|}{ Model 2: policies } \\
\hline Offer help to quit tobacco use & 0.79 & $<0.01$ & 0.40 & 0.37 & 0.03 & 0.30 \\
\hline Warn on dangers of tobacco & -0.38 & 0.07 & & a & a & \\
\hline Anti-tobacco mass media campaigns & a & a & & 0.36 & 0.04 & \\
\hline Raise taxes on tobacco & 0.31 & 0.06 & & 0.36 & 0.04 & \\
\hline \multicolumn{7}{|l|}{ Model 3: socio-economic factors + policies } \\
\hline Conventional cigarette smokers & 0.35 & 0.04 & 0.53 & N.I. & & 0.38 \\
\hline Conventional cigarette ex-smokers & N.I. & & & 0.33 & 0.07 & \\
\hline Gross Domestic Product (GDP) per person & 0.48 & 0.01 & & a & & \\
\hline Offer help to quit tobacco smoke & 0.68 & $<0.01$ & & 0.34 & 0.04 & \\
\hline Warnings on dangers of tobacco & -0.38 & 0.04 & & N.I. & & \\
\hline Anti-tobacco mass media campaigns & N.I. & & & 0.38 & 0.03 & \\
\hline Raise taxes on tobacco & 0.39 & 0.01 & & 0.46 & 0.01 & \\
\hline
\end{tabular}

N.I. not included

${ }^{a}$ Not present at the last step of backward elimination process

The prevalence of ever e-cigarette use was associated with the best practice rankings of the offer of help to quit tobacco consumption [(i.e., cost coverage of nicotine replacement therapy cost covered, availability of a national help-line) $(\beta=0.34 ; P=0.04)$ ], anti-tobacco mass media campaigns $(\beta=0.38 ; P=0.03)$, and with the raise of taxes on tobacco $(\beta=0.46 ; P=0.01)$. None of the other variables investigated were significantly associated with the prevalence of ever e-cigarette use.

\section{Discussion}

The debate on e-cigarettes has escalated in the past few years. In a recent commentary, Martin McKee highlighted three critical gaps in our knowledge about e-cigarettes: are they safe, do they work as a quit aid, and are young people who would not otherwise smoke take up e-cigarettes or conventional cigarettes (McKee 2014). Evidence informing these issues would help researchers and decision-makers clarify the potential of e-cigarettes as a quit aid and whether they should be subject to the same regulations as conventional cigarettes (McKee et al. 2014).

The current investigation is the first European study to provide evidence on country-level correlates of e-cigarette use. Among non-policy variables, the findings suggest that e-cigarette use does not relate to country-level indicators of educational attainment or health, but is associated with the prevalence of current conventional cigarette smoking and with GDP per person. These results are consistent with previous reports showing that former smokers and smokers who tried to quit in the last year are more likely to have used e-cigarettes (Vardavas et al. 2014). Our results, however, contrast with those suggesting that people with higher education attainment are more likely to try e-cigarette (Ramo et al. 2015). Moreover, the international literature suggests that socioeconomic status might be associated with e-cigarette use (Brown et al. 2014).

Importantly, policies controlling conventional tobacco products were associated with both current and ever e-cigarette use. Current e-cigarette use was positively associated with the best practice ranking of national quit smoking services (i.e., cost coverage of nicotine replacement therapy, availability of a national quit line), and the percentage of taxes in the retail price of tobacco products. Higher use of e-cigarettes in countries with higher taxes, and thus higher prices of tobacco products, supports the premise that economic factors play a role in whether people choose to smoke and which products they choose to use (Bader et al. 2011). On the other hand, increased e-cigarette use in countries with higher best practice rankings for national quit smoking services policy, suggests that smokers may try e-cigarette to stop smoking. 
The effectiveness of e-cigarettes in helping smokers quit is highly debated at the international level. Several investigations support their usefulness (Pokhrel et al. 2015; Kaplan 2015a, b; Ben Taleb et al. 2015), while others focus on the lack of evidence on safety and effectiveness in supporting quitting (Levitz 2015a, b). At least one observational study suggests that e-cigarettes support smokers in their quit attempts, (Manzoli et al. 2015) while the randomized controlled trials to date report that "E-cigarettes, with or without nicotine, were modestly effective at helping smokers to quit" (Bullen et al. 2013). Our data suggest that people living in countries with policies that combat tobacco consumption are more likely to smoke or to have ever tried e-cigarettes. We can thus hypothesize that smokers who are more sensitized to tobacco risks across Europe try, or have at least tried in the past, e-cigarettes, which are probably viewed as a helpful way to quit regardless of safety or effectiveness (Vardavas et al. 2014).

Our findings suggest that anti-tobacco mass media campaigns are associated with ever, but not current e-cigarette use. A Cochrane Systematic Review claimed that mass media interventions are useful tools in tobacco control (Bala et al. 2013). Again this evidence suggests that smokers who are aware of tobacco risks may try e-cigarettes to quit smoking.

The only policy that was negatively associated with current e-cigarette use was the warn on dangers of tobacco (health warnings on tobacco products). There is substantial evidence on the effectiveness of pictorial warnings (Fong et al. 2013; Hammond et al. 2013; Mannocci et al. 2013), but relatively little is known about whether such warnings influence e-cigarette use.

Limitations of this study include the ecological design which is subject to the ecologic fallacy, that few covariates at national level were investigated and that the goodness of fit of some models was low (although it was higher in the combined model for both current and ever e-cigarette use).

\section{Conclusion}

Our analysis suggests that both policy and non-policy factors are associated with geographical variability in the prevalence of e-cigarette use. Specifically, among nonpolicy factors, economic factors and the prevalence of conventional cigarette use are directly associated with e-cigarette use. Among tobacco control policies, three were associated with current and ever e-cigarette use including national quit smoking services, mass media campaigns and taxes on the retail price of tobacco products. The presence of health warnings on tobacco products (i.e., pictorial and percentage of packaging covered) was inversely associated with current e-cigarette use. The debate on e-cigarettes is ongoing and there is no consensus on whether they help smokers quit or on the danger to public health (McKee and Capewell 2015). However, the data presented herein suggest that policy makers should be aware that country-level factors may influence e-cigarette use.

\section{References}

Bader P, Boisclair D, Ferrence R (2011) Effects of tobacco taxation and pricing on smoking behavior in high risk populations: a knowledge synthesis. Int $J$ Environ Res Public Health 8(11):4118-4139. doi:10.3390/ijerph8114118

Bala M, Strzeszynski L, Topor-Madry R, Cahill K (2013) Mass media interventions for smoking cessation in adults. Cochrane Database Syst Rev 6:CD004704. doi:10.1002/14651858. CD004704.pub3

Ben Taleb Z, Kenneth W, Asfar T, Bahelah R, Maziak W (2015) Predictors of adherence to pharmacological and behavioral treatment in a cessation trial among smokers in Aleppo, Syria. Drug Alcohol Depend. doi:10.1016/j.drugalcdep.2015.05.038

Bhatnagar A, Whitsel LP, Ribisl KM, Bullen C, Chaloupka F, Piano MR, Robertson RM et al (2014) Electronic cigarettes: a policy statement from the American Heart Association. Circulation 130(16):1418-1436. doi:10.1161/CIR.0000000000000107

Brown J, West R, Beard E, Michie S, Shahab L, McNeill A (2014) Prevalence and characteristics of e-cigarette users in Great Britain: findings from a general population survey of smokers. Addict Behav 39(6):1120-1125. doi:10.1016/j.addbeh.2014.03. 009

Bullen C, Howe C, Laugesen M, McRobbie H, Parag V, Williman J, Walker N (2013) Electronic cigarettes for smoking cessation: a randomised controlled trial. Lancet (London, England) 382(9905):1629-1637. doi:10.1016/S0140-6736(13)61842-5

Database-Eurostat (2015) http://ec.europa.eu/eurostat/data/database. Accessed 24 June 2015

European Commission (2015) Attitudes of Europeans towards tobacco and electronic cigarettes. Special Eurobarometer 429, European Union. doi:10.2875/728026

Famele M, Ferranti C, Abenavoli C, Palleschi L, Mancinelli R, Draisci R (2015) The chemical components of electronic cigarette cartridges and refill fluids: review of analytical methods. Nicotine Tob Res 17(3):271-279. doi:10.1093/ntr/ ntu197

Fong GT, Hammond D, Hitchman A (2013) WHO. The impact of pictures on the effectiveness of tobacco warnings. Bull World Health Organ. http://www.who.int/bulletin/volumes/87/8/09069575/en/

Global Health Observatory Data Repository (2015) WHO. http://apps. who.int/gho/data/node.main.1241?lang=en. Accessed 24 June 2015

Grana R, Benowitz N, Glantz SA (2013) Background paper on e-cigarettes (electronic nicotine delivery systems). Center for Tobacco Control Research and Education, University of California, San Francisco eScholarship. http://escholarship.org/uc/ item/13p2b72n

Gualano MR, Passi S, Bert F, La Torre G, Scaioli G, Siliquini R (2014) Electronic cigarettes: assessing the efficacy and the adverse effects through a systematic review of published studies. J Public Health (Oxf). doi:10.1093/pubmed/fdu055

Hammond D, Geoffrey TF, Borland F, Cummings KM, McNeill A, Driezen P (2013) Text and graphic warnings on cigarette packages: findings from the international tobacco control four 
country study. Am J Prev Med 32(3):202-209. doi:10.1016/j. amepre.2006.11.011

Kadowaki J, Vuolo M, Kelly BC (2015) A review of the current geographic distribution of and debate surrounding electronic cigarette clean air regulations in the United States. Health Place 31:75-82. doi:10.1016/j.healthplace.2014.11.003

Kaplan A (2015a) Can electronic cigarettes assist patients with smoking cessation? Yes. Can Fam Phys 61(6):499-500

Kaplan A (2015b) Rebuttal: can electronic cigarettes assist patients with smoking cessation? Yes. Can Fam Phys 61(6):e255

Levitz S (2015a) Can electronic cigarettes assist patients with smoking cessation? No. Can Fam Phys 61(6):500-501

Levitz S (2015b) Rebuttal: can electronic cigarettes assist patients with smoking cessation? No. Can Fam Phys 61(6):e256

Mannocci A, Colamesta V, Mipatrini D, Boccia A, Terzano C, La Torre G (2013) How would plain packaging and pictorial warning impact on smoking reduction, cessation and initiation? Epidemiol Prev 37(6):400-405

Manzoli L, Flacco ME, Fiore M, La Vecchia C, Marzuillo C, Gualano MR, Liguori G et al (2015) Electronic cigarettes efficacy and safety at 12 months: cohort study. PLoS One 10(6):e0129443. doi:10.1371/journal.pone.0129443

McKee M (2014) Electronic cigarettes: proceed with great caution. Int J Public Health 59(5):683-685. doi:10.1007/s00038-014$0589-\mathrm{Z}$
McKee M, Capewell S (2015) Evidence about electronic cigarettes: a foundation built on rock or sand? BMJ 351:h4863. doi:10.1136/ bmj.h4863

McKee M, Chapman S, Daube M, Glantz S (2014) The debate on electronic cigarettes. Lancet 384(9960):2107. doi:10.1016/ S0140-6736(14)62366-7

Pokhrel P, Herzog TA, Muranaka N, Fagan P (2015) Young adult e-cigarette users' reasons for liking and not liking e-cigarettes: a qualitative study. Psychol Health. doi:10.1080/08870446.2015. 1061129

Ramo DE, Young-Wolff KC, Prochaska JJ (2015) Prevalence and correlates of electronic-cigarette use in young adults: findings from three studies over five years. Addict Behav 4:142-147. doi:10.1016/j.addbeh.2014.10.019

Smith JE (2014) Electronic cigarettes: a safer alternative or potential poison? Home Healthe Nurse 32(9):532-535. doi:10.1097/NHH. 0000000000000138

Vardavas CI, Filippidis FI, Agaku IT (2014) Determinants and prevalence of e-cigarette use throughout the European Union: a secondary analysis of 26566 youth and adults from 27 countries. Tob Contr. doi:10.1136/tobaccocontrol-2013-051394 